gelatinösen Niederschlages aus, der nach kurzer Zeit feinkrystallinisch wird. Das Salz wird abgesaugt, öfters mit kaltem Wasser gewaschen und nachher an der Luft getrocknet. Die Ausbeute beträgt $4.2 \mathrm{~g}$.

Das Dysprosiumkaliumoxalat ist ein weißes, feinkrystallinisches Pulver. In Wasser ist es schwer löslicb, in verdünnten Säuren löst es sich dagegen leicht auf.

$0.5004 \mathrm{~g}$ Sbst.: $0.2171 \mathrm{~g} \mathrm{D}_{5_{2}} \mathrm{O}_{3} .-0.2917 \mathrm{~g}$ Sbst.: $27.1 \mathrm{ccm} \mathrm{KMn} \mathrm{_{4 } -}$ Lösung, $1 \mathrm{ccm} \mathrm{KMn} \mathrm{O}_{4}$-Lösung $=0.043703 \mathrm{~g} \mathrm{C}_{3} \mathrm{O}_{4} \cdot-0.6583 \mathrm{~g} \mathrm{Sbst.:} 0.1382 \mathrm{~g}$ $\mathrm{K}_{2} \mathrm{SO}_{4}-0.3082 \mathrm{~g}$ Sust. verloren bei $180^{\circ} 0.0371 \mathrm{~g} \mathrm{H}_{2} \mathrm{O}$.

$\left[\mathrm{Dy}\left(\mathrm{C}_{3} \mathrm{O}_{4}\right) \mathrm{g}\right] \mathrm{K}+3 \mathrm{H}_{2} \mathrm{O}$. Ber. $\mathrm{D}_{2} \mathrm{O}_{3} 43.2 \mathrm{I}, \mathrm{C}_{9} \mathrm{O}_{4} 40.77, \mathrm{~K} 9.05, \mathrm{H}_{2} \mathrm{O}$ 12.52。 Gef. \43.37, \40.60, $9.42, \gg 12.03$.

Zürich, Chemisches Universitätslaboratorium, April 1911.

173. Giacomo Ciamician und P. Silber: Chemische Lichtwirkungen.

[XIX. Mitteilung.]

(Eingegangen am 6. Mai 1911.)

In unserer 16. Mitteilung ${ }^{1}$ ) behandelten wir einige Versucbe, die sich auf die zwischen Aceton und Methyl- und Äthylalkohol statthabende Einwirkung bezngen. Diese Versuche waren indessen dimals noch nicht ganz abgeschlossen. Eine Ergänzung dieser sozusagen vorläufigen Mitteilungen stellen nun die beute hier mitgeteilten Beobachtungen dar.

\title{
Aceton und Methylalkohol.
}

Diese beiden Körper geben unter dem Einfluß des Licbtes hauptsächlich ein Additionsprodukt, das von Levoli entdeclite Isobutylenglykol,

$$
\begin{aligned}
& \mathrm{CH}_{3} \\
& \mathrm{C}(\mathrm{OH}) \cdot \mathrm{CH}_{2} . \mathrm{OH} \text {. } \\
& \dot{\mathrm{CH}}{ }_{3}
\end{aligned}
$$

Gleicbzeitig bilden sich aber noch andere Verbindungen, die wir im vergangenen Jabre nicht beschrieben haben. Um uns ron dem ganzen Realtionsvorgang Rechenscbaft zu geben, baben wir nun den Versuch nochmals wiederbolt, zum Teil auch unter Aufarbeitung. der früleren Rückstände.

1) B. 43,945 [1910]. 
Das Gemisch von Aceton und Methylalkohol, im Verhältnis von $1: 2$, war während eines ganzen Jahres und $z$ war vom 9. Februar 1910 bis 20. März 1911 belichtet worden. Die farblose, schwach sauer reagierende Flüssigkeit wurde zunächst auf dem Wasserbade destilliert. Der hierbei übergegangene Anteil wurde einerseits nochmals durchgesotten, und so eine um $70^{\circ}$ siedende Fraktion erhalten. Letztere, mit Jod und Phosphor bebandelt, lieferte uns ein Produkt, aus dem wir Isopropyljodia vom Sdp. 88-890 unschwer abscheiden konnten.

Das Aceton batte also, außer daß es sich mit dem Metbylalkohol direkt verband, eine Reduktion zu Isopropylalkohol erlitten. Es blieb uns nur noch übrig, über das Schicksal des Methylalkohols Aufklärung zu erbalten.

Der Rückstand von der Destillation des ursprünglichen Produktes auf dem Wasserbade wies einen geringen Geruch nach Formaldebyd auf. Er wurde mit überschüssigem koblensaurem Kalium ausgesalzen und die abgehobene Ölschicht mit einem Gemisch von Alkohol und Äther ausgezogen. Nach Entfernung der Lösungsmittel und nach wiederholter Destillation des Rückstandes zuerst im Vakuum, dann unter gewöhnlichem Druck, konnten wir, bei $177-180^{\circ}$ übergehend, das Isobutylenglykol abscheiden. Es stellt, der Menge nach, das Hauptprodukt vor. Eine höhere, nicht unbeträchtliche Fraktion sott indessen zwischen $185-200^{\circ}$. Sie enthielt das Äthylenglykol, dessen Siedepunkt bei $197^{\circ}$ ) liegt. Wir haben dasselbe mittels des bei $73^{\circ}$ scbmelzenden $D$ ibenzo ats charakterisiert.

$$
\begin{array}{rl}
\mathrm{C}_{16} \mathrm{H}_{14} \mathrm{O}_{4} \text {. Ber. C } 71.11, \mathrm{H} & 5.19 . \\
& \text { Gef. } 71.36 \text {, } \\
\end{array}
$$

Ein Gemisch von authentischem Dibenzoat mit dem obigen Produkt wies ebenfalls den Scbmp. $73^{\circ}$ auf.

Die Bildung von Äthylenglykol läßt sich unserem Erachten nach in dem Sinne erklären, daß der bei der Reduktion des Acetons auftretende Formaldehyd sich mit dem überschüssig noch vorhandenem Methylalkohol kondensiert:

I. $\mathrm{CH}_{3} \cdot \mathrm{CO} \cdot \mathrm{CH}_{3}+\mathrm{CH}_{3} \cdot \mathrm{OH}=\mathrm{CH}_{3} \cdot \mathrm{CH}(\mathrm{OH}) \cdot \mathrm{CH}_{3}+\mathrm{CH}_{2} \mathrm{O}$. Aceton Methylalkohol Isopropylalkohol Formaldehyd II. $\mathrm{CH}_{3} \cdot \mathrm{OH}+\mathrm{CH}_{2} \mathrm{O}=\mathrm{CH}_{2}(\mathrm{OH}) \cdot \mathrm{CH}_{2} \cdot \mathrm{OH}$. Äthylenglykol

Auch bei der Einwirkung ron Äthylalkohol auf Aceton waren wir in der Lage, einen völlig analogen Vorgang zu beobachten.

1) Beilstein, 3. Aufl., Bd. J, 260. 
Aceton und Äthylalkohol.

Bei unserem vorjährigen Versuch gelang es uns, unter den Produkten der Einwirkung von Äthylalkohol auf Aceton das Würtzsche, bei $177^{\circ}$ siedende Trimethyl-äthylenglykol,

$$
\begin{aligned}
& \mathrm{CH}_{3} \\
& \dot{\mathrm{C}}(\mathrm{OH}) \cdot \stackrel{\mathrm{CH}(\mathrm{OH}),}{\dot{\mathrm{C}}}, \\
& \dot{\mathrm{CH}} \mathrm{CH}_{3}
\end{aligned}
$$

zu erhalten und zu erkennen; aber schon damals deuteten wir auf die Notwendigkeit hin, diese Untersuchung nochmals wieder aufzunehmen, um einen tieferen Einblick in den ganzen Reaktionsverlauf zu gewiunen.

Von vornherein möchten wir bemerken, $d a ß$ in unserer damaligen Mitteilung ein unliebsamer Irrtum beim Zusammenschreiben unterlaufen ist, den wir weiter unten richtig stellen werden.

Ein Gemisch von $1250 \mathrm{ccm}$ Aceton und $2500 \mathrm{ccm}$ Äthylalkohol wurde in zugeschmolzenen Kolben vom 22. April 1910 bis 16. Januar 1911 belichtet. Das Produkt wurde dann zunächst auf dem Wasserbade mit einem Kolonnenapparat destilliert; es fängt bei ungefähr $60^{\circ}$ an iiberzugeben, dann steigt die Siedetemperatur stetig, bis zum Schluß das Thermometer $80^{\circ}$ zeigt. Die zuerst übergebenden Anteile hatten eine lichtgelbe Farbe und einen stechenden, aldehydartigen Geruch. Nachdem wir die Destillation des flüchtigen Anteiles mehrmals wiederbolt und ihn so auf ein kleines Volumen konzentriert batten, erhielten wir eine gelbe, stechend riechende Lösung, aus der wir nach Einwirkung von Hydroxylamin in geringer Menge Krystalle vom Schmp. $244^{\circ}$ (im zugeschmolzenen Rohr bei $236^{\circ}$ ) erhalten konnten. Diese Krystalle sind augenscheinlich identisch mit dem Dioxim des Diacetyls, für welches $\mathrm{F}$ ittig ${ }^{1}$ ) den Schmp. $234.5^{\circ}$ angibt. Das Auftreten des Diacetyls verdankt augenscheinlich seine Herkunit dem Acetaldehyd:

$$
\mathrm{CH}_{3} . \mathrm{COH}+\mathrm{CH}_{3} . \mathrm{COH} \rightarrow \mathrm{CH}_{3} . \mathrm{CO} . \mathrm{CO} . \mathrm{CH}_{3} \text {. }
$$

Wie bei der entsprechenden Reaktion mit Methylalkohol, war auch hier die Gegenwart von Isopropylalkohol zu erwarten. Da nun sein Siedepunkt $\left(82^{\circ}\right)$ wenig verschieden ist von dem des gewöbnlichen Alkobols, baben wir die bei der Destillation im Wasserbade zu allerletzt übergehenden Anteile jodiert; nur so war es uns möglich, das I sopropyljodid vom Schmp. $89.5^{\circ}$ vom Äthyljodid $\left(72.3^{\circ}\right)$ zu trennen. Die Gegenwart des Isopropylalkohols unter den Reaktionsprodukten war somit bewiesen.

Den Rückstand von der Destillation auf dem Wasserbade, der unter vermindertem Druck von den letzten Spuren von Alkohol be-

ग) A. 249, 204. 
freit war, und ca. $100 \mathrm{~g}$ betrug, enthielt das Trimethyl-äthylenglykol, gleichzeitig aber noch andere Körper. Um eine Trennung $z u$ bewerkstelligen, wandten wir folgenden Kunstgriff an: Wir destillierten das Gemisch auf dem Wasserbade im Vakuum unter Zugabe von wenig Wasser, das von Zeit zu Zeit erneuert wurde, wie sich die Flüssigkeit konzentrierte. Auf diese Weise erbielten wir ein wäßriges Destillat, in dem der flüchtige Anteil sich angereichert batte.

Dieses Destillat wurde nun mit kohlensaurem Kalium ausgesalzen; so wurde eine ölige Flüssigkeit erbalten, die unter einem Druck von $13 \mathrm{~mm}$ bei $80-82^{\circ}$ (bei gewöhnlichem Druck bei $177^{\circ}$ ) sott und identisch mit dem im vorigen Jahre erbaltenen ${ }^{2}$ ) Trimethyl-äthylenglykol war.

$$
\begin{array}{ll}
\mathrm{C}_{3} \mathrm{H}_{12} \mathrm{O}_{2} \text {. } & \text { Ber. C 57.69, H } 11.54 . \\
& \text { Gef. \$ 57.78, " } 57.78 .
\end{array}
$$

Die so getrennte Verbindung war indessen noch nicht völlig rein, sondern enthielt noch einen anderen, weniger flüchtigen Körper, der hauptsächlich im Destillationsrückstand sich vorfand; vielleicht auch noch in geringer Menge eine andere Verunreinigung, deren Isolierung uns aber noch nicht gelungen ist. Über die Natur dieses Glykols gab sein Verbalten beim Erbitzen mit verdünnter Schwefelsäure keinen Aufschlu B: wir erhielten bekanntlich im vorigen Jahre hierbei hauptsächlich Methyl-isopropyl-keton.

Der bei der fraktionierten Destillation mit Wasser im Vakuum zurückgebliebene Anteil, eine dickölige, braungefärbte Lösung, wurde seinerseits gleichfalls mit überschüssigem kohlensaurem Kalium versetzt und dann mit Äther ausgezogen. Der Ätherrückstand sott auch nach mehrmaligem Destillieren unter $13 \mathrm{~mm}$ Druck, fast der ganzen Menge nach, bei $81-83^{\circ}$.

Die Analyse des zuletzt übergehenden Ánteiles

$$
\begin{aligned}
& \mathrm{C}_{5} \mathrm{H}_{12} \mathrm{O}_{2} \text {. Ber. C 57.69, } \mathrm{H} 11.54 \text {. } \\
& \mathrm{C}_{4} \mathrm{H}_{10} \mathrm{O}_{2} \text {. Ber. } 53.33 \text {, \ } 11.11 \text {. } \\
& \text { Gef. " 54.92, " } 11.74 \text {. }
\end{aligned}
$$

zeigt, daß derselbe koblenstoffärmer als die entsprechende, aus dem Destillat erbaltene Verbindung war, augenscheinlich aber noch von der letzteren beigemengt enthielt.

1) In unscrer 16. Mitteilung wird dieses Glykol beschrieben als erhalten aus dem Rückstand von der Destillation mit Wasserdampf, während die beiden daselbst mitgeteilten Analysen sich tatsächlich auf ein Produkt beziehen, das ans dem Destillat mit Wasserdampi erhalten worden war. 
Nach der Beobachtung, die wir mit dem Methylalkobol gemacht hatten, schien die Annahme, daB ein Butylenalkohol, $\mathrm{C}_{4} \mathrm{H}_{10} \mathrm{O}_{2}$, entstanden durch Kondensation von Acetaldehyd mit Äthylalkohol, vorliege, nicht unberechtigt.

$\mathrm{Da}$ in diesem Falle wegen des geringen Unterschiedes der Siedepunkte der beiden Glykole an eine Trennung mittels fraktionierter Destillation nicht zu denken war, haben wir zu einem Kunstgriff unsere Zuflucht genommen.

Wir wollen hier gleich bemerken, daß dieser Punkt der Untersuchung unsere Geduld sehr in Anspruch genommen hat: zu verschiedenen Malen sind wir im Verlauf der letzten Jahre auf dieses Thema, obne es zu erledigen, von neuem zurückgekommen, bis scbließlich dann alle Schwierigkeiten glücklich überwunden wurden.

Unter der Voraussetzung, daß von den beiden, im Gemisch vorhandenen Glykolen das Trimetbyläthylenglykol zur Pinakolin-Umlagerung (Methylisopropylketon) mehr Neigung besitze, als das andere, haben wir beide Fraktionen, die leichter und die weniger leicht flüchtige, im Rohr mit verdünnter Schwefelsäure $(1: 10)$ auf $130^{\circ}$ erhitzt. Der Rohrinhalt, der aus zwei Schichten besteht, wird destilliert, bis die ölige obere Schicht übergangen ist. Von diesem Produkt wird weiter unten die Rede sein. Der Destillationsrückstand wurde mit Barythydrat genau abgesättigt; das Filtrat vom schwefelsauren Barium, mit kohlensaurem Kalium ausgesalzen, schied eine obenschwimmende dicke Ölschicht ab, die nit Äther aufgenommen wurde. Der nach Abdunsten des Äthers bleibende Ölrückstand wurde einer öfter wiederbolten, sorglältigen Destillation unterworfen. Unter einem Druck von $11 \mathrm{~mm}$ siedet er zum größten Teil bei $83^{\circ}$, unter gewöbnlichem Druck beobachteten wir $180-184^{\circ}$.

Von diesem Produkt erhielten wir, ausgehend von $20 \mathrm{~g}$ des destillierten Anteils, $4.4 \mathrm{~g}$ (neben $11.7 \mathrm{~g}$ Roh-Methylisopropylketon) und von $19.2 \mathrm{~g}$ des Destillationsrückstandes im Vakuum auf dem Wasserbade $9.2 \mathrm{~g}$ (neben $8.0 \mathrm{~g}$ Metbylisopropylketon).

Die Gegenwart einer weniger flücbtigen Verbindung als das Trimethyläthylenglykol (Sdp. $177^{\circ}$ ) war somit bewiesen.

$$
\begin{aligned}
& \mathrm{C}_{4} \mathrm{H}_{10} \mathrm{O}_{2} \text {. Ber. C 53.33, H } 11.11 \text {. } \\
& \text { Gef. » 54.03, » 11.06, 54.07, } 11.38 \text {. }
\end{aligned}
$$

Obwohl die erbaltenen Zahlen auf die Formel eines Butylenglykols, $\mathrm{C}_{4} \mathrm{H}_{10} \mathrm{O}_{2}$, hinwiesen, waren sie noch nicht hinreichend genau; das Produkt also noch nicht genügend rein.

Um über seine Zusammensetzung und Konstitution Aufklärung zu erbalten, stellten wir die Benzoylverbindung und mit Phenylisocyanat das Phenylurethan-Derivat dar; ferner haben wir es nach dem Vorgang von 
Pechmann ${ }^{1}$ ) mit Bromwasser im Sonuenlicht oxydiert. Wir wollen bier gleich bemerken, daß schon der Siedepunkt es wabrscheinlich machte, da $B$ in unserem Produkt vorwiegend Dimethyl-äthylengly kol enthalten war, denn von den beiden Verbindungen, die aus der Niowirkuug von Acetaldehyd aul Äthylalkohol bätten entstehen können:

$\mathrm{CH}_{3} \cdot \mathrm{CH}(\mathrm{OH}) \cdot \mathrm{CH}(\mathrm{OH}) \cdot \mathrm{CH}_{3}$ und $\mathrm{CH}_{3} \cdot \mathrm{CH}(\mathrm{OH}) \cdot \mathrm{CH}_{2} \cdot \mathrm{CH}_{2} \cdot \mathrm{OH}$, siedet die erstere, wie unsere, bei $183-184^{\circ}$ und die $z$ weite bei $203-204^{\circ}$ ).

Indem wir nun, wie dies Pechman n beim Methyl-äthyl-äthylenglykol ausführte, einen Teil unseres bei $180-184^{\circ}$ siedenden Glykols mit Bromwasser im Sonnenlicht oxydierten, erbielten wir eine gelbe, flüchtige Verbindung von stechendem Geruch, die, mit Hydroxylamin bebandelt, uns das Dioxim des Diacetyls vom Schmp. $240^{\circ}$ lieferte. $\mathrm{C}_{4} \mathrm{~B}_{8} \mathrm{O}_{2} \mathrm{~N}_{2}$. Ber. $\mathrm{N}$ 24.14. Gef. $\mathrm{N} 24.21$.

Auf diese Weise war auch die Gegenwart des Dimetbyläthylenglykols unter den Belichtungsprodukten des Gemisches von Aceton und Ätbylalkobol bewiesen.

Beim Behandeln der Fraktion 180-184 ${ }^{\circ}$ mit Phenylisocyanat auf dem Wasserbade und dem systematischen Krystallisieren der entstandenen Verbindungen aus Benzul erbielten wir schließlich zwei Körper von derselben Zusammensetzung und in nahezu gleicher Menge, von denen der eine, der schwer löslicbe, bei $201-202^{\circ}$, und der andere bei $175^{\circ}$ schmolz.

$\mathrm{C}_{18} \mathrm{H}_{20} \mathrm{O}_{4} \mathrm{~N}_{2}$. Ber. C 65.85, H 6.09, N 8.53.

Schmp. $201-202^{\circ}$

Gef. C 65.77, H 6.45, N 8.68 .

$\gg$ " 66.04, $\$ 6.34$.

$\gg 65.94, \cdot 65$ ?.
Schmp. $175^{\circ}$

Gef. C 65.94, H 6.17, N 8.83.

- $6613, \gg 6.61, \geqslant 8.6 \%$.

$\gg 65.85,>6.74,>8.66$.

Mit Rücksicht auf den Siedepunkt unseres Glykols, das bei 180 $-184^{\circ}$ überging, erscheint die Gegenwart der beiden oben angedeuteten Butylenglykole wenig wahrscheivlich; es ist vielmebr anzunehmen, daß die beiden Pbenyluretbane das racemische und das inaktive Stereoisomere des Dimethyläthylenglykols, das zwei asymmetrische Kohlenstoffatome enthält, darstellen:

$\mathrm{CH}_{3} . \mathrm{CH}\left(0 . \mathrm{CO} . \mathrm{NH} . \mathrm{C}_{6} \mathrm{H}_{5}\right) \cdot \mathrm{CH}\left(\mathrm{O} . \mathrm{CO} \cdot \mathrm{NH} . \mathrm{C}_{6} \mathrm{H}_{5}\right) \cdot \mathrm{CH}_{3}$.

Auch mit Benzoesäureanhydrid, unter Zusatz von benzoesaurem Natrium erbielten wir aus dem bei $180-184^{\circ}$ siedendem Glykol zwei verschiedene Dibenzoate: ein festes, das aus Petroläther in großen,

') B. 23, 2427 [1890].

2) Beilstein, 3. Aufl., I, 262. 
bei $77^{\circ}$ schmelzenden Krystallen herausfällt, und ein flüssiges. Die Zusammensetzung des ersteren:

$$
\begin{aligned}
\mathrm{C}_{38} \mathrm{H}_{18} \mathrm{O}_{4} \text {. } & \text { Ber. C 72.48, H 6.04. } \\
& \text { Gef. 》 72.47, » 6.28. }
\end{aligned}
$$

entspricht der Formel $\mathrm{CH}_{3} \cdot \mathrm{CH}\left(\mathrm{O} . \mathrm{COC}_{6} \mathrm{H}_{5}\right) \cdot \mathrm{CH}\left(\mathrm{O} . \mathrm{COC}_{6} \mathrm{H}_{5}\right) \cdot \mathrm{CH}_{3}$.

Hr. Prof. Giovanni Boëvis, welcher die krystallographische Bestimmung ausführte, teilte uns Folgendes mit:

Krystallsysten: monoklin.

$$
\begin{gathered}
\mathrm{a}: \mathrm{b}: \mathrm{c}=0.4170: 1: 0.3337 . \\
\beta=69^{\circ} 6^{\prime}
\end{gathered}
$$

Beobachtete Formen: $\{110\},\{111\},\{001\},\{010\}$

$\begin{array}{ccccr}\text { Winkel } & \text { Grenzwerte } & \text { Mittel } & \text { ber. } & \text { Nr. } \\ (111):(001) & 48^{\circ} 25^{\prime}-48^{\circ} 48^{\prime} & 48^{\circ} 35^{\prime} & - & 6 \\ (001):(110) & 70^{\circ} 19^{\prime}-70^{\circ} 53^{\prime} & 70^{\circ} 35^{\prime} & - & 10 \\ (110):(110) & 42^{\circ} 31^{\prime}-42^{\circ} 37^{\prime} & 42^{\circ} 34^{\prime} & - & 4 \\ (111):(010) & - & 73^{\circ} 23^{\prime} & 73.13 & 1\end{array}$

Die flüssige Verbindung, die von der eben beschriebenen durch Absaugen getrenut wurde, wurde mehrmals durchgesotten und destillierte der Hauptmenge nach unter einem Druck von $16 \mathrm{~mm}$ bei 217 $-218^{\circ}$. Die Analyse derselben lieferte leider keine guten Resultate:

$$
\begin{aligned}
& \mathrm{C}_{18} \mathrm{H}_{18} \mathrm{O}_{4} \text {. Ber. C 72.48, H 6.04. } \\
& \text { Gef. > 73.81, \$ 5.15. }
\end{aligned}
$$

Das Destillat, von dem weiter oben die Rede war, und das wir neben dem Butylenglykol beim Behandeln des Gemisches von diesem und dem Trimetbyläthylenglykol mit verdünnter Schwefelsäure erbielten, hätte, folgender Gleichung nach ausschließlich aus Methylisopropylketon bestehen sollen:

$$
\left(\mathrm{CH}_{3}\right)_{2} \mathrm{C}(\mathrm{OH}) \cdot \mathrm{CH}(\mathrm{OH}) \cdot \mathrm{CH}_{3}-\mathrm{H}_{2} \mathrm{O}=\left(\mathrm{CH}_{3}\right)_{2} \mathrm{CH} \cdot \mathrm{CO} \cdot \mathrm{CH}_{3} \text {. }
$$

In der Tat ergab eine Probe, die wir mit dem nach Wagner') dargestellten Glykol ausführten, indem wir dasselbe mit Schwefelsäure in Rohr auf $130^{\circ}$ erbitzten, uns ausschließlich das oben erwähnte Keton.

Zu unserem Erstaunen hatte nun aber das von uns untersucbte Destillat keinen einheitlichen Siedepunkt. Der flüchtigere Anteil ging hauptsächlich bei $93-95^{\circ}$ über (Methylisopropylketon siedet bei $93.5^{\circ}$ oder $\left.95^{\circ}\right)$ ), außerden knnnten wir aber noch eine audere Fraktion alsscheiden, die bei $147-150^{\circ}$ sott. Um die das Keton begleitenden Körper zu treunen, bebandelten wir beide Destillate mit Semicarbazid. Jas erste verband sich damit fast vollständig, das zweite hingegen

1) B. 21, 1234 [1888].

3) Beilstein, 3. Aufl., I, 998. 
blieb zum größten Teile ölig, so daß das Öl ohne Schwierigkeit durch Destillation von den Krystallen sich trennen ließ. Letztere bestanden aus dem Semicarbazon des Methylisopropylketons und hatten den Schmp. $\left.112^{01}\right)$. Aus dem Destillat wurde das Öl mit kohlensaurem Kalium ausgesalzen; bei der Destillation sott es glatt bei $149^{\circ}$.

Die Analyse führte zur Formel $\mathrm{C}_{9} \mathrm{H}_{18} \mathrm{O}_{3}$.

$$
\begin{aligned}
& \mathrm{C}_{9} \mathrm{H}_{18} \mathrm{O}_{2} \text {. Ber. C 68.35, H } 11.39 \text {. } \\
& \text { Gef. \68.37, \11.47. }
\end{aligned}
$$

Der Körper enthält keine Äthoxylgruppe und ist permanganatbeständig. Es ist nicht unwahrscheinlich, daß es als ein gemischtes Oxyd der beiden Glykole anzusprechen ist:

$$
\mathrm{C}_{5} \mathrm{H}_{12} \mathrm{O}_{2}+\mathrm{C}_{4} \mathrm{H}_{10} \mathrm{O}_{2}=2 \mathrm{H}_{2} \mathrm{O}+\mathrm{C}_{9} \mathrm{H}_{18} \mathrm{O}_{3} \text {. }
$$

Bezüglich seiuer Herkunft ist nicht auszuschließen, daß es der Behandlung der beiden Glykole mit der verdünnten Schwefelsäure seine Entstehung verdankt, obwohl, wie wir schon oben erwähnten, Trimethyläthylenglykol für sich unter gleichen Bedingungen ausschließlicb Methylisopropylketon liefert.

Aus den eben beschriebenen Versucben geht also herror, daß unter der Einwirkung des Lichtes auf ein Gemisch von Aceton und Äthylalkobol eine Reihe von Reaktionen statthat, die vollständig mit denen beim Methylalkohol beobachteten sich vergleichen lassen. Durch Addition bildet sich Trimethyl-äthylenglykol, wie wir dies voriges $\mathrm{Jahr}$ beschrieben haben; das Aceton erleidet dann auf Kosten des Äthylalkohols eine Reduktion zu Isopropjlalkohol. Der gelegentlich dieser $z$ weiten Reaktion entstandene A cetalde hyd verschwindet fast vollständig aus dem Reaktionsprodukt; zu einem nur geringen Teil wird er wahrscheinlich durch das Aceton zu Diacetyl oxydiert, zum größten Teil kondensiert er sich mit dem unverändert gebliebenen Äthylalkobol, um das Dimethyl-äthylenglykol zu bilden.

Trotz unserer genauen Untersuchung ist natürlich nicht auszuschließen, daß noch andere Körper nebenbei entstanden sind, die wir möglicherweise übersehen haben.

Ob das zuletzt erwähnte Anbydrid $\mathrm{C}_{9} \mathrm{H}_{18} \mathrm{O}_{2}$ durch Belichtung entstanden, ist vorläufig noch eine offene Frage.

Dem Diacetyl würde bei der Reaktion mit dem Methylalkohol das Gl y oxal entsprechen, und es wäre möglich, daß dasselbe in kleiner Menge in dem von uns untersuchten Produkt vorbanden gewesen ist.

1) B. 43,947 [1910].

Berichte d. D. Chem. Gesellschaft. Jahrg. XXXXIV. 


\section{Aceton und Isopropylalkohol.}

Im Gegensatz zu den eben beschriebenen komplizierten Reaktionen ist die unter dem Einfluß des Lichtes auf dieses Gemisch eintretende eine sebr einfache: es bildet sich in diesem Falle nur das Additionsprodukt, das Pinak on.

Ein Gemisch von gleichen Teilen Aceton und Isopropylalkohol, im ganzen $1 / 21$, wurde vom 3. Mai des vergangenen Jahres bis zum Februar belichtet. Die Lösung war völlig farblos geblieben und batte nur eive ganz schwach saure Realtion angenommen. Beim Destillieren auf dem Wasserbade ging das unverändert Gebliebene über; der Rückstand wog $41 \mathrm{~g}$ und sott der Hauptmenge nach $\mathrm{z}$ wischen $170-180^{\circ}$. Dieses Destillat behandelten wir nun, obne es weiter noch zu reinigen, mit der berechneten Menge Wasser. Die beiden Flüssigkeiten mischten sich mit einander unter Erwärmung, und beim Abkühlen erstarrte die ganze Masse $\%$ großen, wohlausgebildeten 'Tafeln. Zwischen Fließpapier abgepreßt, schmolzen sie bei $47^{\circ}$ und batten die Zusammensetzung des Pinakon-lfydrats, $\mathrm{C}_{6} \mathrm{H}_{14} \mathrm{O}_{2}+6 \mathrm{H}_{2} \mathrm{O}$.

$$
\begin{aligned}
& \mathrm{C}_{6} \mathrm{H}_{14} \mathrm{O}_{2}+6 \mathrm{H}_{2} \mathrm{O} \text {. Ber. C 31.86, H } 11.50 . \\
& \text { Gef. } 32.03 \text {, ” } 11.62 .
\end{aligned}
$$

Nach Linnemann ${ }^{1}$ ) hat dasselbe den Scbmp. $46.5^{\circ}$.

Zım Schluß möchten wir hier noch bemerken, daß das Methyläthylketon ein vom Aceton durchaus verschiedenes Verhalten zeigt. Die diesbezüglichen Versuche werden wir in einer der folgenden Mitteilungen beschreiben.

Jas Verbalten des Acetons $z u$ den ersten drei Alkoholen der Fettreihe brachte uns auf den Gedanken, unsere alten Versucbe ${ }^{2}$ ) über Acetophenon und Benzophenon nochmals mit relativ größeren Mengen zu wiederholen. Damals hatten wir beobachtet, daß durch die Einwirkung des Sonnenlichtes der Alkohol zu Acetaldehyd oxydiert wurde, wäbrend entsprechend gleichzeitig eine Reduktion der beiden aromatischen Ketone zu Pinakonen stattfand. Es war unsere Absicht, zu sehen, ob bei dieser Reaktion etwa in kleiner Menge auch eine Addition von Alkohol an die beiden Ketone eintrat.

Fine Lösung von etwa $10 \mathrm{~g} \mathrm{Benz} \mathrm{op} \mathrm{benon} \mathrm{in} 25 \mathrm{ccm}$ Äthylalkohol belichteten wir zu diesem Zweck während des Sommers. Das abgeschiedene Rohpiuakon wog im ganzen $10.1 \mathrm{~g}$. Die alkoholische Lōsung, aus der das Benzopinak on sich abgeschieden hatte, hinterließ beim Abdunsten nur einen geringen harzartigen Rückstand, augenscheinlich herstammend von dem bei der Reaktion entstandenen Aldehyd.

1) Beilstein, 3. Aufl, I, 265.

2) B. 34, 1537 [1901]. 
Benzophenon verwandelt sich also glatt und vollständig in Benzopinakon; bezüglich des Acetophenons war es uns bisher noch nicht möglich, über die Natur des zwischen $80-90^{\circ}$ schmelzenden Körpers Aufklärung zu erhalten.

Hr. Fedro Pirani, der uns bei dieser Arbeit mit großem Fleiße unterstützte, sprechen wir auch an dieser Stelle unseren Dank aus.

Bologna, 4. Mai 1911.

174. Hans Stobbe und Frich Ebert: Die Lichtabsorption elniger korrespondierender Äthan-, Athylen- und AcetylenDerivate.

[Aus dem Chemischen Laboratorium der Universităt Jeeipzig.]

(Eingegangen am 1. Mai 1911.)

Eine demnächst zu publizierende Arbeit über die Beziehungen zwischen Iichtabsorption und Konstitution stereoisomerer Ätbylenund Butadien-Derivate fübrte dazu, auch die diesen Verbindungen korrespondierenden Ätban- und Acetylen-, Butan- und DiacetylenDerivate in gleicher Richtung zu studieren. Die Untersuchung umfaßt die folgenden fünf Reihen:

1. Ätbyl-benzol, Styrol und Phenyl-acetylen.

2. Hydrozimtsäure, die stereoisomeren Zimtsäuren und Phenylpropiolsäure.

3. Benzyl-acetophenon, Benzal-acetophenon und Phenyl-benzoylacetylen.

4. Dibenzyl, Stilben und Tolan.

5. Diphenyl-butan, Diphenyl-butadien und Diphenyl-diacetylen, denen sicb als letztes noch das Diphenyl-butenin, $\mathrm{C}_{6} \mathrm{H}_{5}$. CH:CH.C $: \mathrm{C}$. $\mathrm{C}_{6} \mathrm{H}_{5}$, anschließt.

Trotzdem die Glieder der beiden ersten Reiben teils früher ${ }^{1}$ ), teils gleichzeitig ${ }^{2}$ ) mit uns von anderen Forschern spektroskopisch untersucht worden sind, hielten wir es doch für angezeigt, die bisherigen Messungen unter vollkommen einheitlichen Bedingungen zu wiederholen und uns so bei den Vergleichen mit den von uns neu gemessenen Stoffen unabhängig zu machen von Abweichungen, die bei dieser Methode durch Benutzung verschiedener Apparate und durch verän-

1) Hartley, Baly und ibre Mitarbeiter. Kaysers Handbuch der Spektroskopie, III. Band, und Soc. 93, 1751 und 1902 [1908].

2) Ley und r. Engelhardt, Pb. Ch. 74, 31 and 58 [1910]. 